\title{
Efecto de la retirada de las estatinas durante el ingreso en unidades de Medicina Intensiva
}

\author{
M. Ruiz-Bailén ${ }^{\mathrm{a}, \mathrm{b}}$ \\ a Unidad de Medicina Intensiva, Servicio de Cuidados Críticos y Urgencias, Complejo Hospitalario de Jaén, Jaén, España \\ ${ }^{\mathrm{b}}$ Departamento de Ciencias de la Salud, Universidad de Jaén, Jaén, España
}

Recibido el 11 de octubre de 2009; aceptado el 28 de noviembre de 2009

Disponible en Internet el 22 de enero de 2010

\section{PALABRAS CLAVE \\ Mortalidad; \\ Estatinas; \\ Enfermedad crítica; \\ Accidente \\ cerebrovascular \\ agudo; \\ Sepsis; \\ Síndrome coronario \\ agudo}

\section{KEYWORDS}

Mortality;

Statins;

Critical illness;

Stroke;

Sepsis;

Acute coronary

syndrome

\begin{abstract}
Resumen
Las estatinas se están convirtiendo en uno de los fármacos más utilizados ante la prevención cardiovascular. Dada la edad de los pacientes admitidos en las unidades de medicina intensiva, muchos de ellos reciben tratamiento previo con estatinas. Sin embargo, tras el ingreso en estas unidades suele discontinuarse el tratamiento de estatinas. No existen grandes estudios que evalúen el efecto de la retirada de estatinas sobre la mortalidad. No obstante, los estudios existentes apoyan la hipótesis de que la retirada de estatinas se sigue de un claro efecto perjudicial. Por esto, nos deberíamos replantear de forma muy juiciosa la retirada de las estatinas tras el ingreso en medicina intensiva.
\end{abstract}

(c) 2009 Elsevier España, S.L. y SEMICYUC. Todos los derechos reservados.

Effect of delay in statins during admission in medical units

\begin{abstract}
Statins are becoming one of the drugs used most for cardiovascular prevention. Given the age of the patients admitted to the intensive medicine units, many of them receive previous treatment with statins. However, after admission to these units, statin treatment is generally discontinued. There are no important studies that evaluate the effect of discontinuing statins on mortality. However, the existing studies support the hypothesis that withdrawing statins has a clear harmful effect. Thus, the withdrawal of statins after admission to an intensive medicine unit should be carefully evaluated.
\end{abstract} (c) 2009 Elsevier España, S.L. and SEMICYUC. All rights reserved.

Correos electrónicos: mrb1604@terra.es, ruizbailen@telefonica.net. 
La administración de estatinas es una medida efectiva como prevención primaria y secundaria en la enfermedad cardiovascular, por esto, su uso está ampliamente extendido y aumenta progresivamente. No obstante, cuando estos pacientes presentan un episodio que les ocasiona un ingreso en las unidades de medicina intensiva, no siempre se mantiene su uso. El objetivo de este manuscrito es analizar el posible efecto causado de la retirada de las estatinas sobre los pacientes críticos.

En los pacientes con síndrome coronario agudo (SCA) es indiscutible el efecto beneficioso de la administración de estatinas. De hecho, en registros clínicos como el NRMI ${ }^{1}$, el GRACE $^{2}$ o el PRIMS ${ }^{3}$ se observa un hecho muy interesante, es el beneficio que genera la administración de estatinas durante la fase aguda del infarto agudo de miocardio (IAM), al margen de si la estaban tomando previamente o la inician por primera vez en la fase aguda del IAM. En ambos casos, es decir, la administración de estatinas durante la fase aguda del IAM se asocia a una disminución de la mortalidad. Este hallazgo no ha podido ser contrastado en los ensayos clínicos, pues los ensayos clínicos suelen excluir los pacientes que hubiesen tomado estatinas previamente. Este hallazgo es sumamente interesante, pues significa que la administración precoz de estatinas tras un IAM confiere un efecto protector que podría ser independiente de la administración previa. Al mismo tiempo, algunos estudios encuentran que la discontinuación en la administración de estatinas se asocia a un incremento de mortalidad, con odds ratio (OR) ajustadas que podrían oscilar entre 1,12 (IC del 95\%:1,05-1,20 ${ }^{1}$ y 2,93 (IC del 95\%: $\left.1,64-6,27\right)^{3}$. Estos hallazgos corroboran la hipótesis de que las estatinas tienen un potente efecto cardioprotector, hallazgos que fueron totalmente independientes de los niveles de colesterol.

Aunque parece claro que la retirada de las estatinas en la fase aguda o crítica incrementa la morbimortalidad, se discute el efecto que pudiera tener su retirada sobre pacientes con SCA estables. El ensayo TNT, que evalúa la seguridad y la eficacia de dosis de 10 versus $80 \mathrm{mg}$ al día de atorvastatina, no encuentra diferencias en la discontinuación de las estatinas para la variable combinada de episodios cardiovasculares en los pacientes estables ${ }^{4}$. Daskalopoulou et al evalúan la discontinuación de las estatinas sobre un registro canadiense realizado entre los años 2002 y $2004^{5}$. La inclusión de los pacientes en la cohorte fue a partir del día 90 tras el IAM, y se realizaron 4 grupos: 1) los pacientes que nunca se trataron con estatinas; 2 ) aquellos pacientes que tomaban previamente estatinas y las continuaron tras el IAM; 3) los pacientes que las iniciaron tras el episodio, y 4) los pacientes que suspendieron las estatinas tras el IAM. La cohorte incluyó 9.939 supervivientes del IAM (edad media: $68,4 \pm 12,8$ años; el $60,3 \%$ fueron hombres). Solo en un $22,7 \%$ de los pacientes no fueron prescritas las estatinas tras el IAM. Al considerar el grupo sin administración de estatinas $(n=2.124)$ como referencia, el riesgo relativo (RR) ajustado para la muerte para el grupo $2(\mathrm{n}=2.026)$ fue de 0,84 $(0,66-1,09)$, para el grupo $3(n=5.652)$ fue de 0,72 $(0,57-0,90)$, mientras que para el grupo $4(n=137)$ el RR fue de $1,88(1,13-3,07)$. Shalev et al también encuentran que la retirada de las estatinas, en pacientes estables, se asocia a mayores efectos cardiovasculares ${ }^{6}$. Por lo tanto, la discontinuación de las estatinas, de forma tardía, tras el IAM también puede asociarse con un incremento de la mortalidad. Una explicación posible es que la mayor discontinuación se realiza en las poblaciones con más riesgo y mayor mortalidad, como, por ejemplo, los pacientes más añosos (paradoja de las estatinas), aunque parece claro el beneficio de las estatinas en la población senil ${ }^{7,8}$. Si bien la población de mayor edad, en especial la mayor de 66 años, tiene en un seguimiento a 2 años una adherencia a la administración de estatinas relativamente baja, que oscila entre un $41 \%$ de los pacientes con SCA, un $36,1 \%$ para los pacientes con enfermedad crónica y un $25,4 \%$ para los pacientes para prevención primaria9. Esta falta de adherencia al tratamiento podría infraestimar la eficacia de las estatinas en la población senil. A pesar de que la discontinuación de aspirina, betabloqueantes o estatinas genera un incremento de la mortalidad, ya sea por separado o en conjunto, al menos uno de cada 5 pacientes abandona uno de estos fármacos y uno de cada 8 abandona los 3 fármacos, y se asocia a los no continuadores mayor causa de mortalidad ${ }^{10}$. En la actualidad se estima que la adherencia a las estatinas es al año inferior al $50 \%{ }^{10}$.

La explicación por la que el cese abrupto de las estatinas induce incremento de mortalidad o un aumento de los episodios cardiovasculares es compleja y no bien comprendida. Por un lado, no se puede descartar que parte del beneficio se atribuya a que los pacientes que reciben tratamiento con estatinas (continuadas o iniciadas) formaran un grupo de pacientes «especialmente bien tratados», con más probabilidad de recibir betabloqueantes, IECA, intervencionismo coronario percutáneo, etc., o que fuesen pacientes simplemente seleccionados, pudiendo ser «más jóvenes», o con menor comorbilidad. No obstante, en todos los estudios la regresión logística y el análisis de propensión sitúan que el efecto beneficioso es atribuible a las estatinas, aunque siguen siendo estudios observacionales. Probablemente la explicación más real sea la asunción de un efecto cardioprotector. Este potencial efecto cardioprotector o «pleiotrópico» parece ser múltiple y no está relacionado, al menos inicialmente, con la disminución de los lípidos. En éstos se atribuyen procesos de señalización, diferenciación y proliferación celular ${ }^{11}$. El efecto deletéreo de la retirada de las estatinas podría venir mediado por la retirada del efecto beneficioso $y$, además, por la producción de un efecto rebote $^{11}$. Mediante estudios experimentales se ha demostrado que las estatinas tienen control sobre factores implicados en el desarrollo y en el pronóstico del SCA. Estos efectos incluyen la actividad del óxido nítrico (ON) endotelial, las endotelinas, las metaloproteínas, los efectos antitrombóticos y profibrinolíticos, como inhibición del activador del plasminógeno, el activador tisular del plasminógeno y la génesis de radicales libres. Posiblemente el mecanismo molecular principal de este efecto cardioprotector, no dependiente de la disminución de la lipidemia, sea la inhibición de vías intermedias de los isoprenoides, en la vía de generación del colesterol. Los isoprenoides son esenciales para la función de la señal de transducción de moléculas de la familia «Rho». El efecto de la regulación de la actividad de las moléculas Rho, por las estatinas, es mucho más rápido que el efecto de las estatinas sobre los lípidos $^{3,11}$. Un hecho que refuerza este efecto es la demostración, mediante modelos experimentales, de que la retirada de las estatinas genera una marcada reducción de la producción de ON endotelial, mediada por la actividad 
de las moléculas Rho, aunque sin embargo el nivel de lípidos permanece inalterado durante la fase precoz ${ }^{12}$. Esta disminución de $\mathrm{ON}$ endotelial se genera de forma precoz en las primeras $48 \mathrm{~h}$. Esta reducción de ON genera una contrarregulación de los receptores de la angiotensina ॥ $\mathrm{AT}^{11,12}$, de los receptores de la endotelina $1 \mathrm{y}$ de las moléculas de adhesión inflamatoria, con activación trombótica y liberación de citoquinas. Este beneficio atribuible a la administración de estatinas es muy rápido, pero igualmente reversible, tras la discontinuación del tratamiento, hecho que podría provocar una reactivación inflamatoria en las placas vulnerables. El $90 \%$ de los pacientes incluidos en el registro $\mathrm{PRIMS}^{3}$ se trató con nitroglicerina intravenosa, hecho que podría contrarrestar, al menos en parte, el déficit de producción de $\mathrm{ON}$ endotelial endógeno secundario a la retirada de las estatinas. Los nitratos inducen una vasodilatación, sin embargo, el beneficio del ON endógeno es claramente superior a la administración de nitratos, pues generan una mejoría persistente de la función endotelial, con inhibición de la adhesión de los leucocitos o la inhibición de la activación plaquetaria y de la oxidación del colesterol ligado a lipoproteínas de baja densidad. Sin embargo, la administración de nitroglicerina no genera estos benefi$\operatorname{cios}^{13}$. Otra posibilidad de acción inmediata es el claro rol antiinflamatiorio de las estatinas, e incluso el papel ante la limitación de la lesión posreperfusión coronaria ${ }^{11-14}$. En esta línea, Mensah et $\mathrm{al}^{14}$, mediante un modelo experimental murino, encuentran que el pretratamiento con atorvastatina durante 1, 2 o 3 días ejerce un efecto cardioprotector frente al IAM, pero tras 2 semanas de tratamiento con atorvastatina, se limita este efecto cardioprotector, que podría recapturarse al administrar una dosis suplementaria de atorvastatina durante la fase aguda del IAM. Este efecto lo encuentran ligado a la activación de la vía PI3K/Akt, vía que genera cardioprotección frente a la isquemia y frente a la lesión por reperfusión, promoviendo la supervivencia celular y un efecto antiapoptótico, y dificulta la activación crónica que causa remodelado patológico. El grupo de Pasceri ${ }^{15}$ encuentra en un ensayo clínico resultados similares. Sobre 383 pacientes (edad media de $66 \pm 10$ años, 305 hombres) con angina estable (53\%) o SCA sin elevación del segmento ST (47\%), tratados ambulatoriamente con estatinas (el 55\% con atorvastatina), en los que se realiza ICP; se aleatorizaron a recibir una carga de atorvastatina $(80 \mathrm{mg} / 12 \mathrm{~h}$ antes de la intervención y una dosis posprocedimiento de $40 \mathrm{mg}$ [ $n=192])$ o placebo $(n=191)$. Todos los pacientes recibieron atorvastatina $(40 \mathrm{mg} / \mathrm{día})$. El objetivo primario fue la incidencia de episodios cardiovasculares a los 30 días (muerte cardíaca, IAM o revascularización). Este objetivo primario ocurrió en el 3,7\% del grupo de atorvastatina versus el $9,4 \%$ en el grupo placebo. El análisis multivariado determinó que la dosis suplementaria de atorvastatina era un protector independiente frente al desarrollo del objetivo primario, en especial en el SCASEST. Hallazgos que refuerzan la hipótesis de la necesidad de administración de una dosis alta de estatinas en el momento agudo ${ }^{15}$.

Otra acción interesante es la posibilidad que presentan las estatinas de generar un incremento de células endoteliales progenitoras y un efecto migratorio de estas células ${ }^{16}$; estas células podrían tener un rol importante en la angiogénesis y podrían contribuir a la estabilización de los pacientes con $\mathrm{SCA}^{17}$. También se ha observado un rápido incremento de los niveles de proteína $C$ reactiva tras la retirada de las estatinas y se ha observado que los pacientes que reciben $10 \mathrm{mg}$ diarios de atorvastatina tienen un incremento de la proteína $C$ reactiva de casi el doble al segundo día del cese de las estatinas, sin cambios en los niveles de colesterol, hecho que podría estar en relación con el incremento de los efectos adversos ${ }^{18}$.

Este efecto deletéreo de la discontinuación de estatinas no se ha observado exclusivamente en el SCA, sino que se ha observado en otras enfermedades incluidas en el área de conocimiento de la medicina intensiva ${ }^{19-35}$.

Mediante un estudio observacional que incluía a 631 supervivientes de un accidente cerebrovascular agudo y que se trataron al alta con estatinas ( 322 hombres sin evidencia clínica de enfermedad coronaria, con una edad media de $70,2 \pm 7,6$ años) se observó que la discontinuación de estatinas se mostró relacionada con un incremento de la mortalidad al año. De ellos, 246 pacientes $(38,9 \%)$ abandonaron las estatinas, abandono producido con una media de tiempo desde el alta de 48,6 \pm 54,9 días (mediana 30 días). En el seguimiento fallecieron 116 pacientes. El análisis multivariado mostró que la discontinuación de las estatinas fue un poderoso factor predictor para la mortalidad al año (RR ajustado; IC del 95\%: 2,78 [1,96-3,72]) ${ }^{19}$.

Mediante un ensayo clínico, aleatorizado y controlado que incluía 215 pacientes ingresados por accidente cerebrovascular agudo isquémico, De Blanco et $\mathrm{al}^{20}$ se observó que, de ellos, los 89 pacientes que tomaban previamente estatinas se aleatorizaron a la suspensión de las estatinas durante los 3 primeros días del episodio agudo o a recibir $20 \mathrm{mg} /$ día de atorvastatina de forma precoz. Los pacientes a los que se les retiró la administración de estatinas presentaron una mayor mortalidad o dependencia, RR ajustado de 8,67 $(3,05-24,63)$, y un mayor volumen del infarto. Estos hallazgos concuerdan con los indicados por Singhai et $\mathrm{al}^{21}$, quienes mediante un estudio observacional retrospectivo que incluye 514 pacientes con hemorragia subaracnoidea (HSA) detectan que la administración de estatinas podría generar vasoespasmo, pero al mismo tiempo los 19 pacientes que discontinúan las estatinas podrían tener un mayor riesgo de vasoespasmo cerebral ${ }^{21}$. Aunque Risselada et $\mathrm{al}^{22}$ no encuentran un claro beneficio en la administración indiscriminada de estatinas en 1.004 pacientes con HSA, sí encuentran un hecho muy interesante, y es que el subgrupo de los pacientes que tomaban previamente estatinas y se les suspendieron, presentaron un claro incremento de una nueva incidencia de HSA, por lo tanto, la retirada de estatinas se podría asociar al desarrollo de nueva HSA o resangrado $(O R=2,34$; IC del 95\%: 1,35-4,05). Mediante un ensayo clínico controlado en fase $\|$, de 80 pacientes con HSA, que se aleatorizaron a recibir dosis de $40 \mathrm{mg} /$ día de pravastatina, frente a placebo durante 14 días, se detecta seguridad y eficacia en la administración de estatinas. En este estudio se excluyen los pacientes que previamente habían tomado estatinas. Al final del estudio los pacientes tratados con pravastatina presentaron una menor incidencia de vasoespasmo cerebral y de mortalidad. Otro hecho interesante es que la mejoría neurológica sólo fue significativa en los primeros 14 días, lo que podría explicarse por un empeoramiento tras el abrupto abandono de la pravastatina ${ }^{23}$. Mediante otros ensayos similares, en los que tras confirmar la HSA mediante angiografía, se aleatorizan a 
recibir simvastatina en dosis de $80 \mathrm{mg} / 24 \mathrm{~h}$ versus placebo. A los 14 días la incidencia de vasoespasmo fue menor en el grupo tratado con simvastatina 24,25 .

Además, la retirada de estatinas se ha encontrado, en múltiples estudios observacionales, como un fuerte predictor independiente de mortalidad en los pacientes postoperados de cirugía vascular, y se desaconseja de forma reiterada su cese, dado el incremento de morbimortalidad que conlleva ${ }^{26-28}$. Westphal et $\mathrm{al}^{29}$ mediante un modelo experimental humano encontraron que la administración de fluvastatina en dosis de 40 y $80 \mathrm{mg}$ mejora el flujo, mediado por un efecto de la vasodilatación en la arteria braquial, y esta mejoría ocurría preferentemente en los pacientes con bajo flujo previo, por disfunción endotelial preexistente. No obstante, a las $24 \mathrm{~h}$ de la retirada de la fluvastatina el flujo se deterioraba.

Iguales hallazgos podrían indicarse ante la cirugía cardiovascular. Collard et $\mathrm{al}^{30}$, mediante un estudio longitudinal y prospectivo que incluyó 5.436 pacientes a los que se les realizó cirugía de revascularización coronaria electiva, encontraron una mejoría significativa en la mortalidad global (en los 3 primeros días, un 1,4 versus un 0,3\%; $\mathrm{p}<0,001$ ), aunque no hubo diferencias en la mortalidad hospitalaria. Sin embargo, el análisis multivariante detectó que la suspensión de las estatinas se asoció independientemente con un aumento del riesgo de mortalidad cardíaca postoperatoria (mortalidad cruda del 1,91 versus el $0,45 \%$; $\mathrm{p}<0,01)$. Otros grupos encuentran hallazgos similares ${ }^{31,32}$.

En el área de la medicina intensiva existe una gran controversia sobre el efecto de las estatinas en los pacientes con sepsis. Con este objetivo el grupo de Kruger et $\mathrm{al}^{33}$ evaluaron la mortalidad a los 28 días de una cohorte de 438 pacientes con bacteriemia. De ellos, 66 pacientes $(15 \%)$ recibían estatinas en el momento del ingreso y, de ellos, 56 pacientes $(85 \%)$ continuaron con las estatinas ${ }^{25}$. Las estatinas usadas fueron simvastatina en 36 pacientes, atorvastatina en 22 pacientes y pravastatina en 8 pacientes. Hubo una reducción en todas las causas de mortalidad (el 10,6 versus el 23,1\%, $p=0,022$; OR =0,4; IC del 95\%: 0,17-0,9) y muerte atribuible a bacteriemia (el 6,1 versus el 18,3\%; $\mathrm{p}=0,014 ; \mathrm{OR}=0,06 ; \mathrm{IC}$ al $95 \%: 0,01-0,44)$ en los pacientes que tomaban estatinas. La reducción en todas las causas de muerte se pronunció más en los pacientes que continuaron con las estatinas (OR=0,058; IC del 95\%: 0,008-0,43), lo que podría significar que la discontinuación con estatinas presenta una OR de muerte por bacteriemia de 17,24. Obviamente, es un estudio observacional, con múltiples posibles sesgos, aunque probablemente los pacientes que recibían estatinas lo hacían como prevención cardiovascular $\mathrm{y}$, por lo tanto, presentaban una mayor comorbilidad. No obstante, posiblemente el hallazgo más interesante de este trabajo no sólo sea la generación de la hipótesis de que las estatinas podrían asociarse a una menor mortalidad ante las bacteriemias, sino que su retirada podría asociarse a un incremento de la mortalidad. Las estatinas podrían aportar además un especial beneficio en los subgrupos de mayor gravedad, en cuanto a la prevención del desarrollo de neumonías o incluso la disminución de la mortalidad de estas neumonías. Mediante un estudio de casos y controles, realizado sobre 4.719 pacientes diabéticos que cursaron con neumonía y con 15.322 controles, se observó que el tratamiento activo con estatinas disminuía significativamente la incidencia de neumonías (OR ajustada =0,49; IC del 95\%: 0,35-0,69). Sin embargo, el uso previo de estatinas, considerado como su administración previa un año antes del desarrollo de neumonía, y el no usarlas actualmente no se asoció al descenso del riesgo de desarrollar neumonía $(O R=0,95$; IC del 95\%: 0,663-1,42). La lectura de este estudio es compleja, pues se trata de un estudio con amplias limitaciones y posibles sesgos, pero establece una hipótesis clara, la posibilidad de que mientras se mantiene un tratamiento con estatinas, podría existir una protección frente al desarrollo de neumonías, pero tras su suspensión podría desaparecer este beneficio ${ }^{34}$. De igual modo, la retirada de las estatinas durante la fase aguda de la insuficiencia cardíaca o insuficiencia renal final podría tener un efecto deletéreo ${ }^{32}$.

Este efecto de incremento de la mortalidad ante la discontinuación de estatinas hace que autores como Prinz et $\mathrm{al}^{35}$ se planteen la búsqueda de formulaciones intravenosas de estatinas para los enfermos con intolerancia enteral (para no discontinuar el tratamiento).

A pesar de que la mayoría de los estudios aportados son observacionales, todos coinciden en encontrar un claro beneficio con el mantenimiento de las estatinas durante la fase aguda del episodio crítico. Al margen de las diferentes posibles explicaciones parece claro que la retirada de las estatinas, en especial en situaciones críticas, incrementa la morbimortalidad. Se observa que el mantenimiento de la adherencia a las estatinas podría ser positivo desde el punto de vista coste efectividad. Esto es muy claro, a pesar de la variabilidad del tratamiento terapéutico, ante la enfermedad cardiovascular o incluso la cerebrovascular aguda ${ }^{36-40}$, y menos claro en el resto de enfermedad crítica ${ }^{40}$. Este hecho debería hacer que nos planteásemos muy juiciosamente el motivo de suspensión de las estatinas durante la estancia en las unidades de medicina intensiva.

\section{Bibliografía}

1. Fonarow GC, Wright RS, Spencer FA, Fredrick PD, Dong W, Every N, et al. Effect of statin use within the first $24 \mathrm{~h}$ of admission for acute myocardial infarction on early morbidity and mortality. Am J Cardiol. 2005;96:611-6.

2. Spencer FA, Allegrone J, Goldberg RJ, Gore JM, Fox KA, Granger CB, et al. Association of statin therapy with outcomes of acute coronary syndromes: The GRACE Study. Ann Intern Med. 2004;140:856-7.

3. Newby LK, Kristinsson A, Bhapkar MV, Aylward PE, Dimas AP, Klein WW, et al. Early statin initiation and outcomes in patients with acute coronary syndromes. JAMA. 2002;287:3085-7.

4. McGowan MP, Treating to New Target (TNT) Study Group. There is no evidence for an increase in acute coronary syndromes after short-term abrupt discontinuation of statins in stable cardiac patients. Circulation. 2004;110:2333-5.

5. Daskalopoulou SS, Delaney JA, Filion KB, Brophy JM, Mayo NE, Suissa S. Discontinuation of statin therapy following an acute myocardial infarction: A population-based study. Eur Heart J. 2008;29:2081-3.

6. Shalev V, Chodick G, Silber H, Kokia E, Jan J, Heymann AD. Continuation of statin treatment and all-cause mortality: A population-based cohort study. Arch Intern Med. 2009;169: 260-8.

7. Kalantzi KJ, Milionis HJ, Mikhailidis DP, Goudevenos JA. Lipid lowering therapy in the elderly: Is there a benefit? Curr Pharm Des. 2005;12:3940-5. 
8. Gaw A. The care gap: Underuse of statin therapy in the elderly. Int J Clin Pract. 2004;58 777-5.

9. Jackevicius CA, Mamdani M, Tu JV. Adherence with statin therapy in elderly patients with and without acute coronary syndromes. JAMA. 2002;288:462-7.

10. Ho PM, Spertus JA, Masoudi FA, Reid KJ, Peterson ED, Magid DJ, et al. Impact of medication therapy discontinuation on mortality after myocardial infarction. Arch Intern Med. 2006;166:1842-7.

11. Cubeddu LX, Seamon MJ. Statin withdrawal: Clinical implications and molecular mechanisms. Pharmacotherapy. 2006;26:1286-8.

12. Laufs U, Endres M, Custodis F, Gertz K, Nickenig G, Liao JK, et al. Suppression of endothelial nitric oxide production after withdrawal of statin treatment is mediated by negative feedback regulation of Rho GTPase gene transcription. Circulation. 2000;102:3100-4.

13. Schulz R. Pleiotropic effects of statins: Acutely good, but chronically bad? J Am Coll Cardiol. 2005;45:1292-4.

14. Mensah K, Mocanu MM, Yellon DM. Failure to protect the myocardium against ischemia/reperfusion injury after chronic atorvastatin treatment is recaptured by acute atorvastatin treatment: A potential role for phosphatase and tensin homolog deleted on chromosome ten? J Am Coll Cardiol. 2005;45: 1281-7.

15. Di Sciascio G, Patti G, Pasceri V, Gaspardone A, Colonna G, Montinaro A. Efficacy of atorvastatin reload in patients on chronic statin therapy undergoing percutaneous coronary intervention: Results of the ARMYDA-RECAPTURE (atorvastatin for reduction of myocardial damage during angioplasty) Randomized Trial. J Am Coll Cardiol. 2009;54:555-8.

16. Dimmeler S, Aicher A, Vasa M, Mildner-Rihm C, Adler K, Tiemann $M$, et al. HMG-CoA reductase inhibition (statins) increase endothelial progenitor cells via the PI 3-kinase/Akt pathway. J Clin Invest. 2001;108:391-7.

17. Asahara T, Masuda H, Takahashi T, Kalka C, Pastore C, Silver M, et al. Bone marrow origin of endothelial progenitor cells responsible for postnatal vasculogenisis in physiological and pathological neovascularization. Cir Res. 1999;85: 221-8.

18. Lee KT, Lai WT, Chu CS, Tsai LY, Yen HW, Voon WC, et al. Effect of withdrawal of statin on C-reactive protein. Cardiology. 2004;102:160-6.

19. Colivicchi F, Bassi A, Santini M, Caltagirone C. Discontinuation of statin therapy and clinical outcome after ischemic stroke. Stroke. 2007;38:2652-7.

20. Blanco M, Nombela F, Castellanos M, Rodríguez-Yáñez M, GarcíaGil M, Leira R, et al. Statin treatment withdrawal in ischemic stroke: A controlled randomized study. Neurology. 2007;69: $900-4$.

21. Singahl AB, Topcuoglu MA, Dorer DJ, Ogilvy CS, Carter BS, Koroshetz WJ. SSRI and statin use increases the risk for vasospasm after subarachnoid hemorrhage. Neurology. 2005; 64:1003-8.

22. Risselada R, Straatman H, Van Kooten F, Dippel DW, Van der Lugt A, Niessen WJ, et al. Withdrawal of statins and risk of subarachnoid hemorrhage. Stroke. 2009;40:2885-7.

23. Tseng MY, Czosnyka M, Richards H, Pickard JD, Kirkpatrick PJ. Effects of acute treatment with pravastatin on cerebral vasospasm, autoregulation, and delayed ischemic deficits after aneurysmal subarachnoid hemorrhage: A phase ॥ randomized placebo-controlled trial. Stroke. 2005;36 1627-1627.

24. Lynch JR, Wang H, McGirt MJ, Floyd J, Friedman AH, Coon AL, et al. Simvastatin reduces vasospasm after aneurysmal subarachnoid hemorrhage: Results of a pilot randomized clinical trial. Stroke. 2005;36:2024-6.

25. Chou SH, Smith EE, Badjatia N, Nogueira RG, Sims $2^{\text {nd }} J R$, Ogilvy CS, et al. A randomized, double-blind, placebo-controlled pilot study of simvastatin in aneurysmal subarachnoid hemorrhage. Stroke. 2008;39:2891-3.

26. Biccard BM, Pooran RR. Validation of a model to predict allcause in-hospital mortality in vascular surgical patients. Cardiovasc J Afr. 2008;19:303-8.

27. Schouten O, Hoeks SE, Welten GM, Davignon J, Kastelein JJ, Vidakovic R, et al. Effect of statin withdrawal on frequency of cardiac events after vascular surgery. Am J Cardiol. 2007;100:310-6.

28. Le Manach Y, Godet G, Coriat P, Martinon C, Bertrand M, Fléron $\mathrm{MH}$, et al. The impact of postoperative discontinuation or continuation of chronic statin therapy on cardiac outcome after major vascular surgery. Anesth Analg. 2007;104:1323-6.

29. Westphal S, Abletshauser C, Luley C. Fluvastatin treatment and withdrawal: Effects on endothelial function. Angiology. 2008;59:613-8.

30. Collard CD, Body SC, Shirnan SK, Wang S, Mangano DT. Preoperative statin therapy is associated with reduced cardiac mortality after coronary artery bypass graft surgery. J Thorac Cardiovasc Surg. 2006;132 392-0.

31. Rostagno C. Recent developments in pharmacologic prophylaxis of atrial fibrillation in patients undergoing surgical revascularization. Cardiovasc Hematol Agents Med Chem. 2009;7:136-7.

32. Laufs U, Custodis F, Böhm M. Who does not need a statin: Too late in end-stage renal disease or heart failure? Heart. 2008;94:1130-8.

33. Kruger $P$, Fitzsimmons $K$, Cook $D$, Jones $M$, Nimmo $G$. Statin therapy is associated with fewer deaths in patients with bacteraemia. Intensive Care Med. 2006;32:75-9.

34. Van de Garde EM, Hak E, Souverein PC, Hoes AW, Van den Bosch JM, Leufkens HG. Statin treatment and reduced risk of pneumonia in patients with diabetes. Thorax. 2006;61:951-7.

35. Prinz V, Endres $M$. The acute (cerebro)vascular effects of statins. Anesth Analg. 2009;109:572-4.

36. Ruiz Bailén $M$. Administración de estatinas durante la fase aguda del síndrome coronario agudo. Med Intensiva. 2009. doi:10.1016/j.medin.2009.07.012.

37. Civeira Murillo E, Del Nogal Sáez F, Álvarez Ruiz AP, Ferrero Zorita J, García Alcántara A, Aguado AG, et al. Med Intensiva. doi:10.1016/j.medin.2009.07.010.

38. Colmenero Ruiz M, Reina Toral A, Jiménez Quintana MM, Aguayo de Hoyos E, Ruiz Bailén M, García Alcántara A, et al. Grupo ARIAM. Variabilidad en el manejo del infarto agudo de miocardio en el registro ARIAM en el año 2002. Med Intensiva. 2005;29:420-9.

39. Rosell Ortiz F, Mellado Vergel FJ, Ruiz Bailén M, García Alcántara A, Reina Toral A, Arias Garrido J, et al. Med Intensiva. 2007;31:502-9.

40. Daskalopoulou SS. When statin therapy stops: Implications for the patient. Curr Opin Cardiol. 2009;24:454-60. 